\title{
SIW 2D PLANAR ARRAY WITH FOUR CROSS SLOTS RADIATOR AND TUNING VIAS
}

\author{
P. Sanchez-Olivares, J. L. Masa-Campos*, J. A. Ruiz-Cruz, \\ and B. Taha-Ahmed
}

Group of Radio-Frequency, Circuits, Antennas and Systems

(RFCAS), Department of Electronic and Communication Technologies, Autonoma University of Madrid, Francisco Tomas y Valiente 11, Madrid 28049, Spain

\begin{abstract}
A Substrate Integrated Waveguide (SIW) planar array is presented using a right handed circularly polarized (RHCP) element with four crossed tilted radiating slots. In addition, a pair of metallic tuning vias is included to really improve the reflection of longest slots. A corporate feeding network over SIW has been designed for distributing the input signal to 128 radiating elements, divided into 8 progressive wave linear arrays of 16 elements each. The designed planar array has been manufactured and measured to verify the antenna performance. $25.5 \mathrm{~dB}$ gain, $2.33 \mathrm{~dB}$ axial ratio, as well as $85 \%$ radiation efficiency values have been experimentally achieved at $17 \mathrm{GHz}$. A $3 \%$ usable bandwidth $(16.75-17.25 \mathrm{GHz})$ is obtained due to the typical frequency main beam tilt dispersion in the elevation plane of the progressive wave arrays.
\end{abstract}

\section{INTRODUCTION}

During the last decades, Substrate Integrated Waveguide (SIW) technology has been consolidated as an alternative to conventional waveguides in millimeter and microwave applications [1-3]. SIW can be manufactured with a printed circuit board (PCB) process preserving the transmission characteristics of waveguides. Furthermore, SIW can be easily connected to planar feed schemes such as microstrip lines within the same substrate [4].

On the other hand, slots are widely used as radiating elements in waveguide array antennas in the whole microwave range [4-9].

Received 9 April 2013, Accepted 10 May 2013, Scheduled 23 May 2013

* Corresponding author: Jose Luis Masa-Campos (joseluis.masa@uam.es). 
Likewise, high gain slot planar arrays fed with waveguide networks have been proposed in [8] and [9] with satisfactory results. In the last years, many authors have characterized several kinds of single slot elements to obtain different radiation features and better performances in array configurations. Montisci presented an element formed by two inclined slots in [2], Chen et al. introduced a four slot element based on a duplicate two slot radiator in [3], and Simmons used crossed slots in [5]. Even more, fractal slot elements have been used for some researches [6]. Therefore, the antennas fabricated on SIW provide as good characteristics as those fabricated on conventional waveguides.

In this paper, a Circularly Polarized SIW Slotted Planar Array (CP-SIWSPA) in $\mathrm{Ku}$ band is designed, simulated and manufactured. Some examples of $\mathrm{Ku}$-band operating systems are the new DBS band for broadcasting downlink $(17.3-17.7 \mathrm{GHz})$, or satellite telemetry and tele-command subsystems $(16.6-17.1 \mathrm{GHz})$. A mixed corporative (azimuth plane) and progressive (elevation plane) feeding network is included, and the slotted radiator behavior presented by authors in [10] is validated in a $2 \mathrm{D}$ array structure. Section 2 presents the antenna design process including the principles of the single element, the mutual coupling model and the SIW power divider. Section 3 shows the simulated and measured array results. Finally, Section 4 summarizes the conclusions.

\section{ANTENNA DESIGN}

\subsection{Single Element - Four Cross Slots with Tuning Vias}

The single radiating element, shown in Figure 1, is formed by four crossed slots [10]. Two $45^{\circ}$ inclined and orthogonal radiating slots can be used to obtain circular polarization [2]. A separation of $\lambda_{g} / 4$ ( $\lambda_{g}$ is the SIW wavelength) between $+45^{\circ}$ and $-45^{\circ}$ slots generates a $90^{\circ}$ phase difference between their corresponding linear $E$-field components. Therefore, circular component is achieved. A reflection cancelling slot is also incorporated to each radiating slot, as indicated in [3]. Likewise, the proposed structure provides the same coupling levels than the inclined slot pair element [2], but with a significant dimension reduction (around 15\%).

The propagated travelling wave from port 1 is coupled according to the slot dimensions, providing right-hand circular polarization (RHCP). All parameters shown in Figure 1 have been optimized to good slot performance in terms of reflection and axial ratio response using CST Microwave Studio [10].

In order to improve the obtained radiating slot reflection coefficient, a pair of metallic tuning via holes is placed beside the 


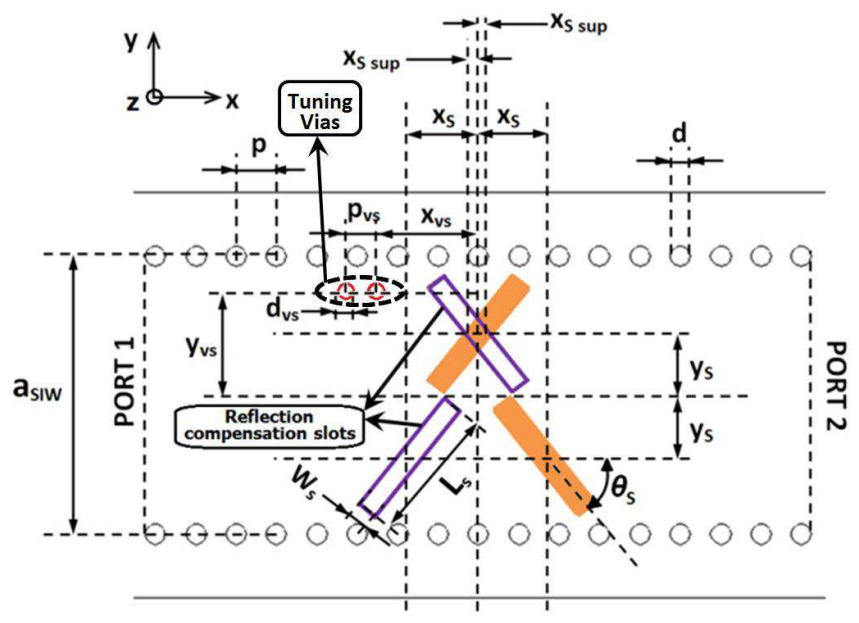

Figure 1. Circularly polarized radiator composed of four cross slots in Substrate Integrated Waveguide with tuning metallized vias.

four slot element, as Figure 1 shows. These tuning vias introduce a capacitive effect which compensates the inductive behavior of the longer slotted elements. Likewise, the coupling element capability is not disturbed for the tuning vias insertion (Figure 2, [10]).

\subsection{Planar Array Design}

\subsubsection{Design of Array Last Element as Matched Load}

In [10] a 16 element progressive wave RHCP linear array antenna with $-26 \mathrm{~dB}$ Taylor distribution and a $2 \%$ residual power (dissipated in a coaxial load at the end of the array) was presented. In this paper, a corporative SIW network is included to feed the 2D planar array, which is compound by 8 progressive wave linear arrays as the one shown in [10]. Nevertheless, as a difference, in this occasion all the input power is fully radiated. Therefore, the last element of each progressive linear array must be designed as a matched load to couple and radiate all the residual power at the end of the SIW array antenna. This new configuration, improve the 2D array performance in terms of dimensions, weight and cost.

Different slot terminations have been developed in the state of art [11]. In this case, the four cross slots with tuning vias of Figure 1 and an ending short-circuit of vias are used as last element in the progressive linear array (Figure 2(a)). The single element parameters 


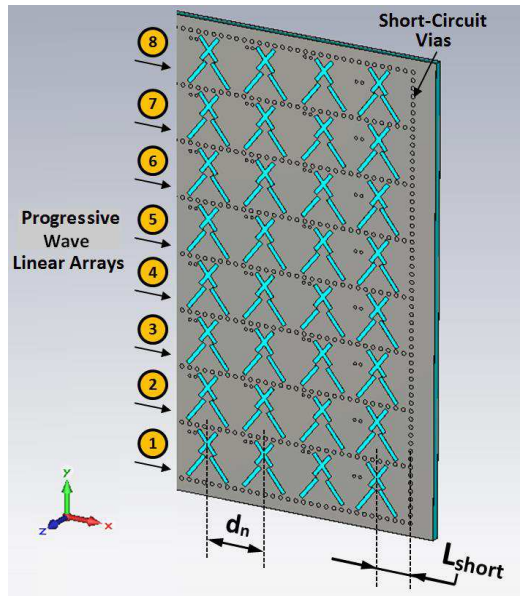

(a)

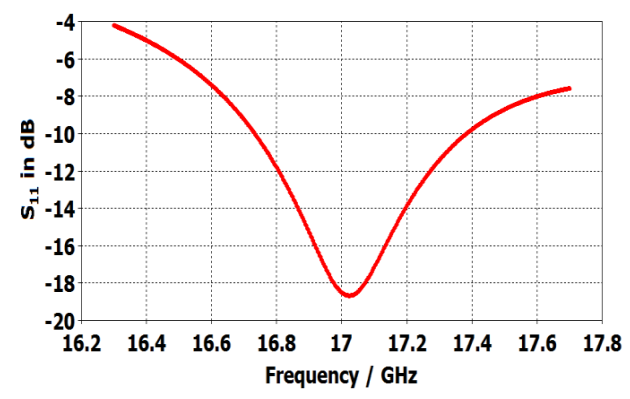

(b)

Figure 2. Progressive wave linear arrays: (a) structure scheme and (b) last element simulated reflection coefficient $\left(L_{s}=5.77 \mathrm{~mm}\right.$, $\left.x_{s}=2.33 \mathrm{~mm}, x_{v s}=2.9 \mathrm{~mm}, y_{v s}=1.6 \mathrm{~mm}, L_{\text {short }}=7.30 \mathrm{~mm}\right)$.

and the separation to the final short-circuit $L_{\text {short }}$ are optimized. As shown in Figure 2(b), the reflection coefficient is under $-10 \mathrm{~dB}$ from 16.7 to $17.4 \mathrm{GHz}$, and the residual wave inside the SIW is entirely radiated.

\subsubsection{Mutual Coupling Model}

The mutual coupling effects between adjacent elements originate degradation of the radiation pattern in terms of beam-width, sidelobe level increase and beam-tilt error. Therefore, the evaluation of mutual coupling between adjacent slots is required for the design of the planar array. The iterative model applied in this paper is similar to the one presented in [12], where authors considered near $E$-field monitors in front of each radiating element. In this paper, a base cell of $3 \times 3$ elements is used (Figure 3 ). By evaluating and comparing the near $E$-field monitors with the theoretical amplitude and phase values ( $-26 \mathrm{~dB}$ Taylor distribution), the central element of the base cell is resized to compensate the mutual effects of the surrounding slots.

Table 1 shows the designed antenna parameters. The separation between $n$-th and $(n+1)$-th elements $(d n)$ has been calculated using the method in [2] (Section 3) to obtain broadside array radiation. 
Table 1. Configuration of SIW slot array antenna.

\begin{tabular}{|c|c|c|c|c|c|}
\hline Index & $\mathbf{L}_{\mathbf{s}} \mathbf{( \mathbf { m m } )}$ & $\mathbf{x}_{\mathbf{s}} \mathbf{( \mathbf { m m } )}$ & $\mathbf{x}_{\mathbf{v s}} \mathbf{( \mathbf { m m } )}$ & $\mathbf{y}_{\mathbf{v s}} \mathbf{( \mathbf { m m } )}$ & $\mathbf{d}_{\mathbf{n}} \mathbf{( \mathbf { m m } )}$ \\
\hline $\mathbf{1}$ & 2.95 & 3.51 & - & - & 14.21 \\
\hline $\mathbf{2}$ & 3.65 & 3.45 & - & - & 13.94 \\
\hline $\mathbf{3}$ & 4.15 & 3.37 & - & - & 13.65 \\
\hline $\mathbf{4}$ & 4.50 & 3.25 & - & - & 13.37 \\
\hline $\mathbf{5}$ & 4.75 & 3.17 & - & - & 13.05 \\
\hline $\mathbf{6}$ & 5.00 & 3.04 & - & - & 12.74 \\
\hline $\mathbf{7}$ & 5.15 & 2.93 & - & - & 12.64 \\
\hline $\mathbf{8}$ & 5.30 & 2.77 & 3.15 & 4.60 & 12.24 \\
\hline $\mathbf{9}$ & 5.41 & 2.63 & 2.90 & 4.55 & 12.15 \\
\hline $\mathbf{1 0}$ & 5.5 & 2.50 & 2.70 & 4.30 & 12.07 \\
\hline $\mathbf{1 1}$ & 5.57 & 2.46 & 2.65 & 4.05 & 11.89 \\
\hline $\mathbf{1 2}$ & 5.61 & 2.45 & 2.65 & 4.00 & 11.84 \\
\hline $\mathbf{1 3}$ & 5.64 & 2.42 & 2.60 & 3.90 & 11.86 \\
\hline $\mathbf{1 4}$ & 5.52 & 2.51 & 2.70 & 4.20 & 12.20 \\
\hline $\mathbf{1 5}$ & 5.36 & 2.69 & 2.80 & 4.50 & 11.46 \\
\hline $\mathbf{1 6}$ & 5.77 & 2.33 & 3.50 & 1.60 & - \\
\hline
\end{tabular}

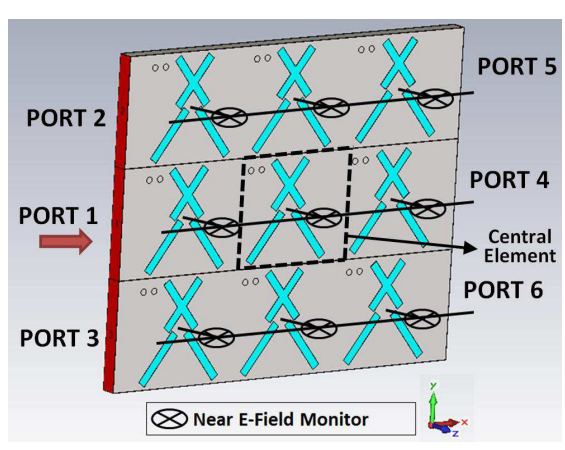

Figure 3. $3 \times 3$ elements structure for mutual coupling model with near $E$-field monitors.

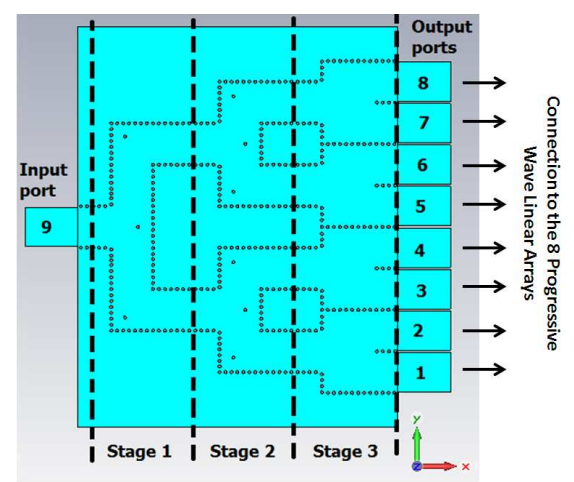

Figure 4. SIW power divider composed by T-junctions and Yjunctions.

\subsection{SIW Corporative Feeding Network}

Three-stage of 2-way dividers have been grouped to shape an 8way power divider over SIW, as Figure 4 shows, to feed the array for the azimuth plane. The first and second stages consist of 
conventional T-junctions with an inductive via as matching post within the corner structure [13]. Nevertheless, the third stage is compound of conventional Y-junctions [14]. Inductive posts are used at the corners to help the wave travel fluently and reduce the return loss. In this paper design, the width of the SIW power divider paths and the width of the SIW progressive wave linear arrays are both $11 \mathrm{~mm}$.

The symmetric configuration guarantees all transmission coefficients equal in terms of amplitude and phase. Figure 5 presents the simulated results of the proposed feeding structure. It can be seen a good reflection response in the operation bandwidth.

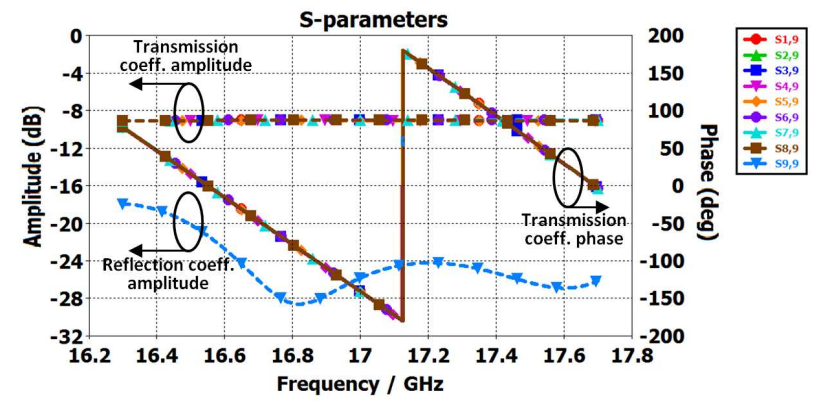

Figure 5. $S$-parameters simulation of SIW power divider (amplitude and phase).

\section{SIMULATION AND EXPERIMENTAL RESULTS}

The CP-SIWSPA has been simulated using the EM commercial software CST Microwave Studio. Figure 6 shows the manufactured

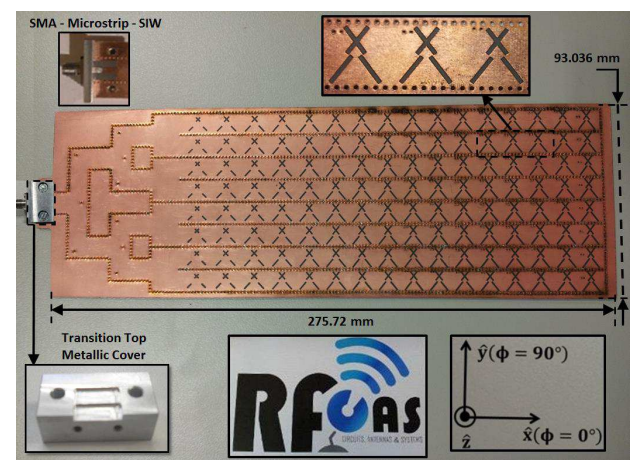

Figure 6. $\mathrm{CP}$ tuning vias $\mathrm{SIW}$ slotted 2D array prototype (CPSIWSPA). 
CP-SIWSPA prototype with TLY-5A TACONIC dielectric substrate $\left(\varepsilon_{r}=2.17, \tan \delta=0.0011\right.$, thickness $\left.=1.57 \mathrm{~mm}\right)$. A SMA-microstripSIW horizontal transition has been used to feed the antenna. An inner mechanized aluminum piece covers the microstrip to SIW taper transformer in order to avoid undesired transition radiation [15].

As Figure 7(a) demonstrates, good agreement between simulated and measured reflection coefficients of CP-SIWSPA is observed. A broad reflection coefficient bandwidth (around 12\%) is achieved under $-10 \mathrm{~dB}$ criteria.

The simulated and measured CP-SIWSPA radiation patterns at the elevation $\left(\phi=0^{\circ}\right.$, according to Figure 6 axes $)$ and azimuth

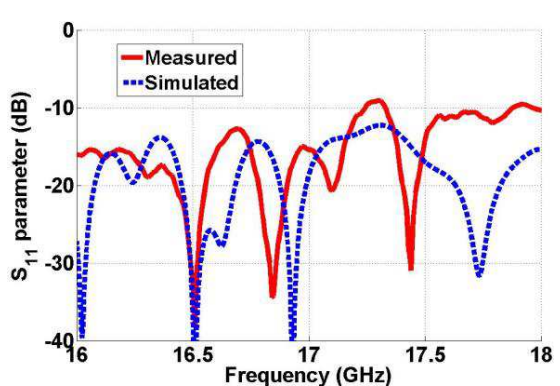

(a)

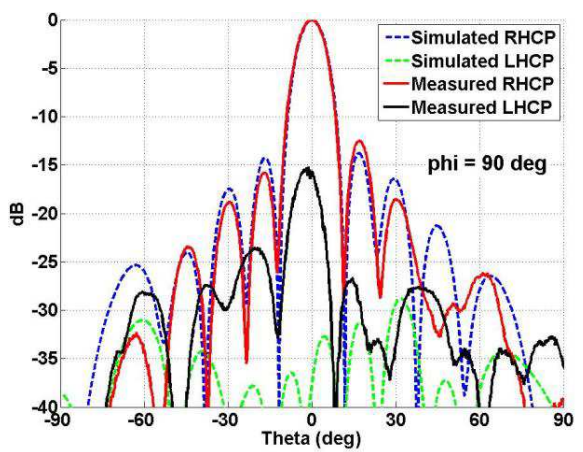

(c)

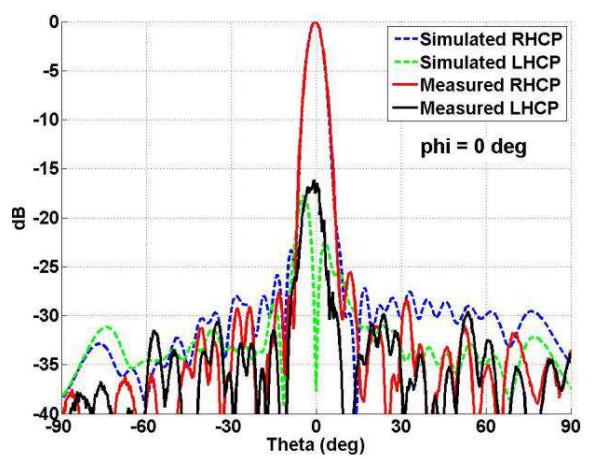

(b)

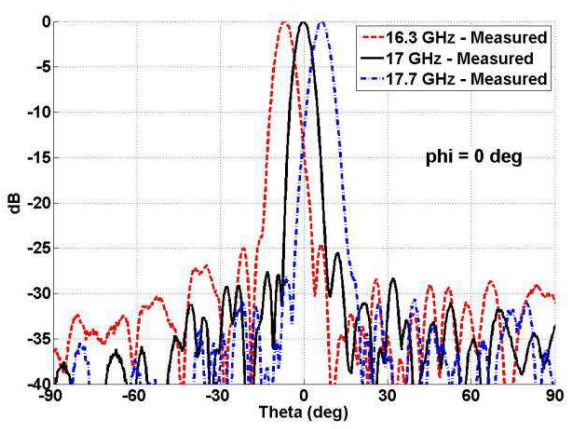

(d)

Figure 7. Measured versus simulated CP-SIWSPA electrical parameters: (a) reflection coefficient, (b) normalized elevation radiation pattern at $17 \mathrm{GHz}\left(\phi=0^{\circ}\right.$ plane), (c) normalized azimuth radiation pattern at $17 \mathrm{GHz}\left(\phi=90^{\circ}\right.$ plane $),(\mathrm{d})$ normalized elevation radiation patterns ( $\mathrm{RHCP})$ at $16.3,17$ and $17.7 \mathrm{GHz}\left(\phi=0^{\circ}\right.$ plane). 
$\left(\phi=90^{\circ}\right)$ planes are shown in Figures $7(\mathrm{~b})-(\mathrm{c})$ respectively. The broadside performance is very well achieved at the design array frequency $(17 \mathrm{GHz})$ for the elevation plane (Figure $7(\mathrm{~b})$ ). Likewise, a $5.6^{\circ}$ value of the $-3 \mathrm{~dB}$ beamwidth, $-25.5 \mathrm{~dB}$ side-lobe level and a $-17.49 \mathrm{~dB}$ LHCP/RHCP ratio in broadside direction have been measured. On the other hand, a $10^{\circ}$ value of the $-3 \mathrm{~dB}$ beamwidth, $-12.5 \mathrm{~dB}$ side-lobe level and a $-16.65 \mathrm{~dB}$ LHCP/RHCP ratio have been measured for the azimuth plane (Figure $7(\mathrm{c})$ ). In both cases, a satisfactory agreement between measured and simulated RHCP components is achieved. However, a LHCP level increase is observed at the main beam angle due to ground plane edge effects, as well as tolerance manufacturing errors. The elevation plane antenna pattern is achieved due to the 8 progressive wave linear arrays. The typical main beam frequency scanning response of this kind of arrays is verified in Figure $7(\mathrm{~d})$. Main beam tilt angles at 16.3 and $17.7 \mathrm{GHz}$ are $-6.5^{\circ}$ and $6.5^{\circ}$, respectively. Therefore, the usable antenna bandwidth is reduced to $3 \%(16.75-17.25 \mathrm{GHz})$, under $\mathrm{a}+/-2.5^{\circ}$ beam dispersion in frequency band over broadside direction [3]. The use of the corporative SIW power divider avoids this effect in the azimuth plane.

The simulated and measured antenna gain at main beam angle, as well as the radiation efficiency response, calculated as the ratio between the simulated and measured gain, are shown in Figure 8. 25.5 dBi gain and $85 \%$ efficiency peak values have been measured at $17 \mathrm{GHz}$. The maximum efficiency (87\%) is achieved at $17.25 \mathrm{GHz}$.

Figure 9 presents the simulated and measured CP-SIWSPA axial ratio (AR) responses in the main beam tilt direction, which verifies

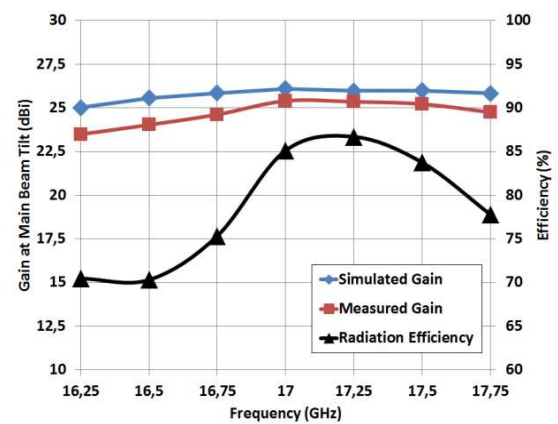

Figure 8. Measured versus simulated gain, and measured efficiency of the antenna prototype at the main beam angle.

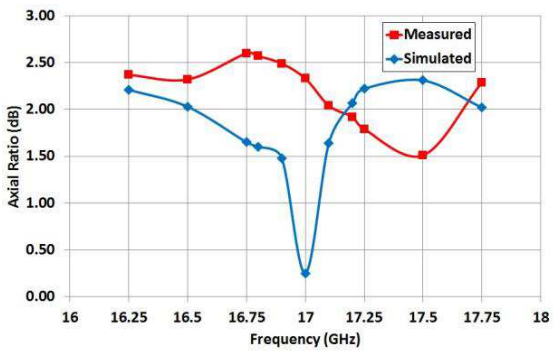

Figure 9. Measured versus simulated antenna axial ratio at the main beam angle. 
the LHCP level increase in Figure 7. The peak measured AR value is $2.33 \mathrm{~dB}$ at $17 \mathrm{GHz}$.

\section{CONCLUSION}

In this paper, a CP SIW planar array with four cross slots and tuning vias radiator has been designed, simulated and fabricated to validate the antenna performance. A corporative SIW divider network is used to obtain broadside and uniform pattern in azimuth plane. The SIW divider feeds 8 progressive wave linear arrays of 16 elements each, broadside pattern and $-26 \mathrm{~dB}$ Taylor distribution for the elevation plane. A mutual coupling compensation model based on near $E$-field monitors has been implemented. Although satisfactory concordance between measured and simulated reflection coefficient or radiation pattern results have been obtained in a wide frequency range (16.3 to $17.7 \mathrm{GHz}$ ), the usable bandwidth is reduced to $3 \%$ due to the main beam tilt frequency dispersion in the elevation plane pattern. A $2.33 \mathrm{~dB}$ axial ratio, $25.5 \mathrm{dBi}$ gain and $85 \%$ radiation efficiency at $17 \mathrm{GHz}$ in broadside direction have been experimentally obtained.

\section{REFERENCES}

1. Yan, L., W. Hong, G. Hua, J. Chen, K. Wu, and T. J. Cui, "Simulation and experiment on SIW slot array antennas," IEEE Microwave Wireless Compon. Lett., Vol. 14, No. 9, 446-448, Sep. 2004.

2. Montisci, G., "Design of circularly polarized waveguide slot linear arrays," IEEE Trans. on Antennas and Propagat., Vol. 54, No. 10, 3025-3029, Oct. 2006.

3. Chen, P., W. Hong, Z. Kuai, and J. Xu, "A substrate integrated waveguide circularly polarized slot radiator and its linear array," IEEE Antennas and Wireless Propag. Letters, Vol. 8, 120-123, 2009.

4. Bakhtafrooz, A., A. Borji, D. Busuioc, and S. Safavi-Naeini, "Novel two-layer millimeter-wave slot array antennas based on substrate integrated waveguides," Progress In Electromagnetics Research, Vol. 109, 475-491, 2010.

5. Simmons, A., "Circularly polarized slot radiators," IEEE Trans. on Antennas and Propagat., Vol. 5, 31-36, Jan. 1957.

6. Trinh-Van, S., H. B. Kim, G. Kwon, and K. C. Hwang, "Circularly polarized spidron fractal slot antenna arrays for 
broadband satellite communications in Ku-band," Progress In Electromagnetics Research, Vol. 137, 203-218, 2013.

7. Masa-Campos, J. L., S. Klinger, and M. Sierra-Pérez, "Parallel plate patch antenna with internal rectangular coupling patches and $\mathrm{TE}_{\mathrm{N} 0}$ mode excitation," IEEE Trans. on Antennas and Propagat., Vol. 57, No. 7, 2185-2189, Jul. 2009.

8. Sekretarov, S. and D. M. Vavriv, "A wideband slotted waveguide antenna array for SAR systems," Progress In Electromagnetics Research M, Vol. 11, 165-176, 2010.

9. Chen, X.-P., K. Wu, L. Han, and F. He, "Low-cost high gain planar antenna array for $60-\mathrm{GHz}$ band applications," IEEE Trans. on Antennas and Propagat., Vol. 58, No. 6, 2126-2129, Jun. 2010.

10. Sánchez-Olivares, P. and J. L. Masa-Campos, "Slot radiator with tuning vias for circularly polarized SIW linear array," 2012 6th European Conference on Antennas and Propagation (EUCAP), Mar. 2012.

11. Hirokawa, J., K. Sakurai, M. Ando, and N. Goto, "Matching-slot pair for a circularly polarized slotted waveguide array," IEE Proc. H, Vol. 137, No. 6, 367-371, 1990.

12. García-Valverde, D., J. L. Masa-Campos, P. Sánchez-Olivares, B. Taha-Ahmed, and J. Córcoles-Ortega, "Linear patch array over substrate integrated waveguide for Ku band," IEEE Antennas and Wireless Propag. Letters, No. 99, Jan. 2013.

13. Hirokawa, J., K. Sakurai, M. Ando, and N. Goto, "An analysis of a waveguide T junction with an inductive post," IEEE Transactions on Microwave Theory and Techniques, Vol. 39, No. 3, 563-566, Mar. 1991.

14. Lin, S., S. Yang, A. E. Fathy, and A. Elsherbini, "Development of a novel UWB vivaldi antenna array using SIW technology," Progress In Electromagnetics Research, Vol. 90, 369-384, 2009.

15. Cheng, J.-C., E. S. Li, W.-F. Chou, and K.-L. Huang, "Improving the high-frequency performance of coaxial-to-microstrip transitions," IEEE Transactions on Microwave Theory and Techniques, Vol. 59, No. 6, Jun. 2011. 